Versuch IV.

Hund Körpergewicht $7000 \mathrm{~g}$.

\begin{tabular}{|c|c|c|c|}
\hline Datu & & ahl der Erythrocyten & Zahl der Leucocyten \\
\hline 12. & & 6660000 & 10000 \\
\hline \multirow[t]{2}{*}{19.} & & 6720000 & 10000 \\
\hline & Subkutane & e Injektion von & 0,06 g Phenylhydrazin \\
\hline 20. & I. & 6540000 & $\begin{array}{l}\text { I2 } 000 \\
0,06 \text { g Phenylhydrazin }\end{array}$ \\
\hline I. & I. & 4900000 & $\begin{array}{l}\text { I I 00o } \\
0.062 \text { g Phenylhydrazin }\end{array}$ \\
\hline 2. & I. & 4920000 & $\begin{array}{l}8000 \\
0,063 \text { g Phenylhydrazin }\end{array}$ \\
\hline 3. & I. & 3890 & I 6000 \\
\hline 4. & I. & 3640000 & 14000 \\
\hline 26. & I. & 3920000 & $r_{4} 000$ \\
\hline 7. & I. & 3280000 & I 5000 \\
\hline 28. & I. & 3940000 & 15000 \\
\hline o. & I. & 2680000 & I 3000 \\
\hline 3 I. I & I. & 2540000 & 9700 \\
\hline
\end{tabular}

Entnahme von $30 \mathrm{ccm}$ Blut und Reinjektion des defibrinierten und präparierten Blutes: $\mathrm{I} 3 \mathrm{ccm}$ intravenös, $10 \mathrm{ccm}$ intramuskulär.

$\begin{array}{lll}\text { I. II. } & 3800000 & \text { I } 3000 \\ \text { 2. II. } & 3160000 & \text { I3 } 000 \\ \text { 3. II. } & 3100000 & 2 \text { I } 000 \\ \text { 4. II. } & 3220000 & \text { I2 } 500\end{array}$

Versuch abgebrochen.

$\mathrm{Zu}$ den vorstehenden Versuchen ist noch zu bemerken, da $B$ die Tiere immer im ganzen pro Kilo Körpergewicht $0,035 \mathrm{~g}$ Phenylhydrazin erhielten, und daß immer auch die jeweils entnommene Blutmenge pro Kilo Körpergewicht $4 \mathrm{~g}$ entsprach. Gelegentlich starben auch Tiere schon bei dieser Dosierung des Phenylhydrazins, noch ehe mit der Blutbehandlung begonnen werden konnte. Es ruft also jedenfalls die gewählte Dosierung des Phenylhydrazins eine sehr schwere Erkrankung bei den Tieren hervor. Immer aber war eine günstige Beeinflussung durch die Autobluttransfusion deutlich nachweisbar.

Anhangsweise will ich noch erwähnen, daß ich auch versucht habe, die Wirkung der Wundhormone am überlebenden Gewebe der Milz nach der bekannten Carreischen Versuchsanordnung nachzuweisen. Diese Versuche sind indessen noch nicht abgeschlossen, so daß ich darüber noch nicht berichten kann. Es bedarf vor allem hier noch einer Verbesserung der Methodik zu dem besonderen Zwecke.

\section{WIRKUNGEN VON TURNEN UND SPORT AUF DIE KÖRPERBILDUNG ERWACHSENER JUNGER MÄNNER.}

\section{Von}

\section{Dr. med. Herbert HerXheimer.}

Aus der Preußischen Polizeischule für Leibesubungen in Spandau.

Je mehr das Interesse der großen Offentlichkeit für Leibesübungen jeder Art wächst, um so mehr ist es Pflicht des Arztes, den Einfluß der Leibesübungen auf den Körper zu erforschen. Denn nur die Kenntnis der normalen Wirkung, welche die Leibesübungen auf den Körper ausüben, befähigt zur Beurteilung dessen, was bereits als schädlich bezeichnet werden muß.

Der Stand unserer Kenntnisse auf diesem Gebiet ist noch sehr gering. Es gibt zwar eine ganze Reihe von Beobachtungen, die über die Einwirkung bestimmter Sportarten gemacht sind, meist an verhältnismäßig kleinem Material. Mit exakten Messungen gehen jedoch die wenigsten einher. Hier ist an erster Stelle $K_{O L B}{ }^{1}$ ) zu nennen. Ein Vergleich des Einflusses verschiedener Arten sportlicher Betätigung auf denselben Menschen findet sich aber nirgends und ein abgerundetes Bild von der Einwirkung körperlicher Tätigkeit ist schon deshalb nicht zu erwarten, weil sich die Arbeiten meist nur mit einer - mehr oder weniger einseitigen Sportart befassen.

Beobachtungen an großem Material, ohne Berücksichtigung eines bestimmten einseitigen Sportzweiges, sind bisher nur an Jugendlichen gesammelt worden.

So hat Matrhias ${ }^{1}$ ) in der Schweiz während eines Jahres junge Männer beobachtet, deren Alter zu Anfang I8-Jahre betrug. Er teilte sie in 2 Gruppen ein. Die erste hatte $4 \frac{1}{2}$ Monate, die zweite I Jahr und 7 Monate am Turnunterricht teilgenommen. Die Gruppe II war nun $z u$ Beginn des Beobachtungsjahres in bezug auf die körperliche Entwicklung, soweit sie in den Körpermaßen zum Ausdruck kommt, voraus. Im Beobachtungsjahre selbst war die Zunahme beider Gruppen ziemlich die gleiche, nur in bezug auf die Zunahme der Oberarmmuskulatur war bei der Gruppe I ein größerer Fortschritt zu verzeichnen.

GodIN ${ }^{2}$ ) beobachtete Schüler im Alter von $x_{4} 1 / 2-I 8$ Jahren. Er sah, daß die Turner unter ihnen in den dreieinhalb Jahren der Beobachtungsperiode größere Fortschritte in der körperlichen Entwicklung machten als die Nichtturner. Während dieser Zeit führte er von I4 Schülern, die bisher nicht am Turnunterricht teilgenommen hatten, 7 dem Turnen zu und fand, daß diese den Abstand, den sie von den Turnern hatten, sprungweise einholten, während die andern 7 weiter zurückblieben.

Die Untersuchungen, die ich $^{3}$ ) im Jahre I920 an Jüng- lingen im Durchschnittsalter von etwa $17^{1 / 2}$ Jahren anstellte, haben die Godins schen Ergebnisse vollkommen bestätigt, obwohl sie sich nur auf volkstümliches Turnen hauptsächlich Laufen - bezogen. Es ergibt sich also aus diesen Arbeiten ganz deutlich, daß die Leibesübungen auf den jugendlichen, in rascher Entwicklung befindlichen Organismus einen lebhaften Wachstumsanreiz auszuüben vermögen.

Es lag nun nahe, den Einfluß auf den erwachsenen Körper zu untersuchen. Uber sein Verhalten fehlen bisher exakte Angaben.

An der Preußischen Polizeischule für Leibesübungen bot sich mir Gelegenheit, darüber Beobachtungen zu sammeln. An dieser Schule werden jüngere Beamte zu Sport- und Turnlehrern für die Polizei ausgebildet. Ihre Ausbildungszeit ist kurz bemessen etwa 3 Monate - und so kommt es, da $\beta$ die Anforderungen, die an ihren Körper gestellt werden, recht erhebliche sind.

Die Beamten wohnten zusammen, hatten alle genau den gleichen Dienst, dieselbe Verpflegung; damit waren die günstigsten äußeren Bedingungen gegeben. Ihre Ausbildung war eine äußerst vielseitige. Sie umfaßte Gerätturnen, Leichtathletik (Laufen, Springen, Werfen), Schwerathletik (Gewichtheben, Ringen). Djiu-Djitsu, Boxen, Schwimmen und Rasenspiele. Die Gesamtanstrengung eines Tages war gerade so bemessen, daß ihre Vermehrung auf die Dauer nicht ohne Schaden hätte exfolgen können. Aus diesem mannigfaltigen Programm ergibt sich, daß von der Einwirkung einer besonderen Sportart hier nicht gesprochen werden kann. Bei unseren Feststellungen kann es sich nur um die Wirkungen vielseitiger körperlicher Betätigung handeln.

Diese Wirkung konnte eine verschiedene sein, je nachdem der Betreffende schon vorher irgendwelchen Sport getrieben hatte oder nicht. Deshalb wurde vor Beginn jedes Kurses genau festgestellt, was der einzelne vorher betrieben hatte und in welchem Umfange das geschehen war. Im ganzen gelangten 2 Kurse zur Beobachtung, einer von etwa go Köpfen, der von Juni bis September, und einer von etwa 60 Köpfen, der von Oktober bis Dezember I92 I lief; jeder Kurs dauerte $x$ I-ri Wochen.

Genaue Untersuchungen fanden zu Beginn, in der Mitte und am Schlusse jedes Lehrgangs statt. Untersucht wurde die Körperlänge, das Gewicht, der Brustspielraum, der Zustand der Muskulatur und der inneren Organe, insbesondere von Herz und Lungen. Das Herz wurde bei einem Drittel der Lehrgangsteilnehmer jedesmal orthodiagraphiert. Die Muskelumfänge wurden am Oberarm und am Oberschenkel gemessen, und zwar in der Mitte zwischen Acromion und Olecranon, bzw. zwischen Trochanter maior und Kniegelenkspalt. Das Durchschnittsalter der Kursteilnehmer betrug 25,03 Jahre in Kurs I und 26,80 Jahre in Kurs II. Ihre Größe, die sich nur ganz unwesentlich veränderte, war durchschnittlich $171,8 \mathrm{~cm}$ in Kurs $I$ und $I 73,5$ in Kurs II, blieb also etwas über dem für dies Alter nach VIERORDT anzunehmenden Mittel von etwa $169 \mathrm{~cm}$.

1) MATTHIAS, Der Einfluß der Leibesübungen auf das Körperwachstum. Jahrbuch đ. Schweiz. Ges. f. Schulgesundheitspfl. I\%, I9I6. zit. Körper und Geist, I918, S. 18. 2) GODIN, La Croissanice pendant l'âge scolaire. Neuchatel t9r3. Coll. d'actua* lités pédagogiques publiée sous les auspices de I'institut J. J. Rousseau. $Z$ it. wie oben.

3) HERXHEIMER, Wirkungen leichtathletischen Sommertrainings auf die körperliche Entwicklung von Jünglingen. Virchows Arch. 233, x92. 
Auch die übrigen Werte waren etwas höher als das Mittel, da es sich um lauter gesunde und wohlgebaute Männer handelte.

\section{A. Beobachtungen über das durchschnittliche Verhalten aller Kursteilnehmer.}

Betrachten wir zunächst die Veränderungen, denen alle Kursteilnehmer im Laufe der Zeit ausgesetzt waren, so dürfen wir uns nur an die Durchschnittswerte halten; auf die maximalen und minimalen Einzelabweichungen können wir weniger Wert legen: sie sind $\mathrm{zu}$ sehr von der individuellen Disposition und von kleinen Verschiedenheiten des Milieus abhängig.

\section{Das Körpergewicht.}

Das Körpergewicht nahm in den ersten Wochen der Kurse durchschnittlich stark zu. Wenn man bedenkt, daß

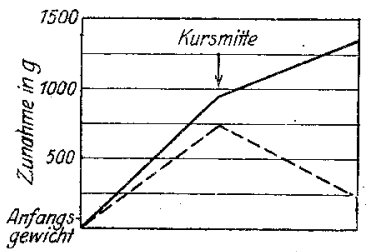

Kurve A I: Gewicht. Kurs I Anfangswert $65,8 \mathrm{~kg}$ .... " II . , $66,6 \mathrm{~kg}$

0,40 kg, also weit weniger um $0,40 \mathrm{~kg}$, also weit weniger Kurs II kam es sogar zu einem Abfall von durchschnittlich $0,49 \mathrm{~kg}$, so daß in Kurs I schließlich eine durchschnittliche Zunahme von $\mathrm{r}, 36 \mathrm{~kg}$, in Kurs II dagegen nur eine von $0,26 \mathrm{~kg}$ resultierte.

Wie haben wir uns diesen Verlauf der Gewichtskurve (Kurve A I) zu erklären? Die Zunahme ist offenbar auf vermehrten Stoffansatz zurückzuführen. Dieser Stoffansatz dürfte sich hauptsächlich auf die Muskulatur erstreckt haben, was sich auch aus den Zunahmen der Muskelumfänge ergibt, die weiter unten beschrieben sind. Denn eine Speicherung von Fett oder Wasser kommt aus verschiedenen Gründen nicht in Frage, die bei einer früheren Untersuchung dargelegt worden $\sin \mathrm{d}^{1}$ ).

Am nächstliegenden ist es natürlich, den Grund des Stoffansatzes in einer gesteigerten Nahrungsaufnahme zu suchen, die ihrerseits wieder durch die vermehrte körperliche Betätigung bedingt ist. Nun war die gebotene Verpflegung zwar gut und reichlich, aber ging durchaus nicht über das Maß dessen hinaus, was den Beamten an ihren bisherigen Standorten zur Verfügung gestanden hatte. Allerdings war das Bedürfnis nach Nahrung außerhalb der gemeinsamen Mahlzeiten gesteigert und wurde vielfach durch Aufnahme von SüBigkeiten (Schokolade) und Milch befriedigt. Aber diese verhältnismäßig sehr unerhebliche Mehrzufuhr kann nicht allein als Ursache für die starke Gewichtszunahme angesprochen werden; dazu war sie zu gering. Es scheint eher, daß durch die körperliche Betätigung irgendwie eine bessere Ausnutzung der gebotenen Nahrung ermöglicht wird. Daß die Gewichtszunahme keine zufällige sein kann, ergibt sich schon aus ihrem Verlauf: jedesmal im Anfang des Kurses äußerte sich die starke Reaktion des Körpers auf die Änderung der bisherigen Lebensweise durch plötzliche Gewichtszunahme. Dann stellte sich der Körper auf die Milieuveränderung ein und die Reaktion wurde schwächer bzw. es trat ein geringer Rückschlag ein.

Obwohl der Verlauf der Gewichtskurven in beiden Kursen grundsätzlich einander ähnlich ist - erst starkes Ansteigen, dann Nachlassen - besteht doch ein quantitativer Unterschied. $\mathrm{Zu}$ seiner Erklärung muß eine weitere Tatsache angeführt werden. Während des zweiten Kurses wurde das Gewicht jede. Woche einmal unter genauen Kautelen - frühmorgens am gleichen. Wochentage und in nüchternem $\mathrm{Zu}$ stand - festgestellt. Bereits nach 3 Wochen war eine Durch-

1) HERXHEIMER, Wirkungen leichtathletischen Sommertrainings auf die körperliche Entwicklung von Jünglingen. Virchows Arch. 233. Igzrr. schnittszunahme von $0,87 \mathrm{~kg}$ erreicht. Bis dahin war der Herbst warm und schön gewesen. Dann schlug die Witterung um und die Temperatur ging erheblich herunter. Es kam hinzu, daß die Aufenthaltsräume der Beamten in den ersten Tagen nicht geheizt werden konnten - ein Teil zog sich leichte Erkältungskrankheiten $\mathrm{zu}$ - und nun zeigte sich bei der nächsten Wägung ein plötzliches Stillstehen der Zunahme, dann ein allmählicher Abfall unter gelegentlichen Remissionen, der bis zum Schlusse des Kurses im Dezember anhielt.

Diese Tatsache läBt den Schluß wohl berechtigt erscheinen, daß der größere Rückschlag und die infolgedessen geringere Gesamtzunahme im. Winterkurs auf die niedrigere Außentemperatur zurückzuführen ist. Es ist ja auch leicht erklärlich, daß der Körper im Sommer bei hoher AuBentemperatur mehr Material zum Stoffansatz verwenden kann als im Winter, wo er einen größeren Teil dieses Materials zu Erhaltung seiner Eigentemperatur verbrauchen muß. Die niedrige Außentemperatur kam bei unsern Kursteilnehmern besonders zur Wirkung, weil die Ausbildung im Sommer wie im Winter in der gleichen dünnen Sportkleidung (kurze Hose und dünnes Baumwolltrikot) vor sich ging.

Freilich soll nun hiermit nicht gesagt ${ }^{-}$sein, daß] die Außentemperatur die alleinige Ursache für die verschiedene Gewichtszunahme in beiden Kursen sei. Unter den anderen Faktoren, welche hierbei möglicherweise eine Rolle spielen, ist in erster Linie das Alter der Versuchspersonen anzuführen. Wie oben erwähnt, ist das Durchschnittsalter in den beiden Kursen um fast zwei Jahre verschieden, Das fällt sehr ins Gewicht, zumal bei vorgerückterem Alter der Stoffansatz infolge körperlicher Anstrengung offenbar nicht mehr mit derselben Leichtigkeit vor sich geht. Wenigstens scheint dies aus der gesonderten Beobachtung der älteren Kursteilnehmer hervorzugehen, die weiter unten beschrieben ist. Daß das Alter aber auch nicht allein Ursache des Unterschiedes sein kann, geht schon daraus hervor, daß auch nach Aussonderung der 9 über 30 Jahre alten Lehrgangsteilnehmer die Durchschnittszunahme des Kurs II immer noch wesentlich hinter der des Kurs I zurückbleibt; sie beträgt o,42 gegenüber I, $36 \mathrm{~kg}$.

\section{Muskelumfänge.}

In beiden Kursen war eine Zunahme der Muskelumfänge sowohl am Oberarm als auch am Oberschenkel zu verzeichnen. Teilweise war diese Zunahme recht beträchtlich; sie erreichte

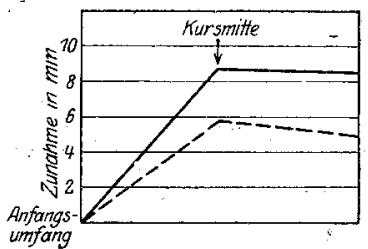

Kurve A II: Oberarmumfang. \begin{tabular}{ll} 
Kurs I Anfangswert $28,6 \mathrm{~cm}$ \\
\hline , II
\end{tabular}

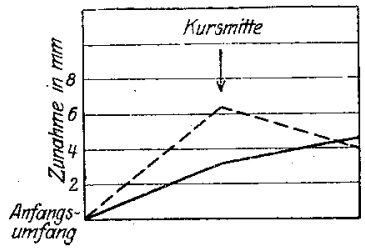

Kurve A II: Oberschenkelumfang Kurs I Anfangswert $49,8 \mathrm{~cm}$
II $, \quad 49,0 \mathrm{~cm}$ in Kurs I am Oberarm 3\% des Umfanges. Der Verlauf der Kurven (A II) entspricht auch im Rhythmus der Zunahme ganz.dem Verlauf der Gewichtsveränderung in beiden Kursen. Erst rapider Zuwachs, dann in der zweiten Kurshälfte Nachlassen oder kleiner Rückschlag. Auch der graduelle Unterschied zwischen beiden Kursen ist wieder vorhandenl. In Kurs II sind die Abnahmen in der zweiten Hälfte stärker und damit die.Gesamtzunahmen geringer. Die Vermehrung der Oberschenkel- und Oberarmumfänge ist wohl mit Sicherheit auf Vergrößerung der.Muskelmenge zurückzuführen, und wir gehen wohl nicht fehl, wenn wir die beobachtete Gewichtszunahme in engsten ursächlichen Zusammenhang damit bringen. Schon der gleiche Rhythmus in der Veränderung beider Kurven"spricht dafür. Eine andere Beobachtung, die ebenfalls für den engen Zusammenhang ins Feld geführt werden muß, sei hier noch mitgeteilt: $Z$ u einer besonderen Untersuchung, über die an anderer. Stelle berichtet ist ${ }^{1}$ ),

1) FIERXHEIMER, Zur Wirkung von prim. Natriumphosphat auf die körperlìche Leistungsfähigkeit. Klin. Wochenschr. Ig22, Nr. ro, S. 480 . 
mußten die Teilnehmer an Kurs II in zwei Gruppen eingeteilt werden, deren Durchschnittsgewicht etwa gleich war. Als dies geschehen war und die durchschnittlichen Muskelumfänge beider Gruppen berechnet wurden, stellte sich heraus, $\mathrm{da} B$ auch die durchschnittlichen Umfänge von Oberarm und Oberschenkel bei beiden Gruppen bis auf $1 / 2 \mathrm{~mm}$ genau gleich waren.

\section{Brustspielraum.}

Das Verhalten des Brustspielraums, des Unterschieds zwischen inspiratorischem und exspiratorischem Brustumfang, bietet in beiden Kursen ein völlig übereinstimmendes Bild. (Kurve A III). In der ersten Hälfte

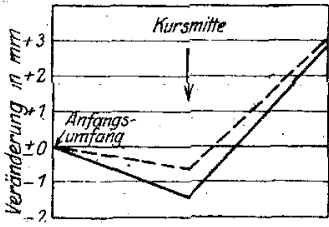

Kurve A III: Bristspielraum. - Kurs I Anfangswert $7,4 \mathrm{~cm}$ sehen wir eine geringe Verkleinerung des Spielraums, die in der zweiten Hälfte von einem immerhin erheblichen Ansteigen gefolgt ist. Als Gesamtresultat finden wir am Ende beider Kurse eine etwa gleich bedeutende Vergrößerung des Brustspielraums, die fast $4 \%$ erreicht. $\mathrm{Zu}$ der gleichen Zeit also, zu der die Muskulatur starken Zuwachs zeigt, geht der Brustspielraum zurück, und wenn der Körper sich in der zweiten Hälfte auf die größere Muskelarbeit eingestellt hat und die Gewichts- und Muskelzunahme nicht mehr mit der anfänglichen Geschwindigkeit vor sich geht, dann steigt der Brustspielraum wieder an und über das Anfangsmaß hinaus.

Wie haben wir nun dieses Phänomen zu erklären?

Es liegt die Annahme nahe, daß der gleiche Faktor; der für das stärkere Muskelwachstum verantwortlich zu machen ist, nämlich die erhöhte körperliche Tätigkeit, auch auf die Größe des Brustspielraums einen Einfluß ausübt. KNOLL $^{1}$ ) hat bei Skiläufern unmittelbar nach großer Anstrengung (Wettlauf) ebenfalls Verkleinerung des Brustspielraums gesehen und dies auf Ermüdung der Atemmuskeln infolge der starken Anstrengung zurückgeführt. Diese Erklärung erscheint ganz einleuchtend, und es soll hier mangels eines Beweises oder Gegenbeweises nicht näher auf ihre Berechtigung eingegangen werden. Denkbar wäre es auch, daß es infolge der starken plötzlichen Beanspruchung der Lunge durch Anstrengung zu einem Zustand akuter (im KNoLL schen Falle) oder subakuter (in unserem Falle) Lungenblähung kommt, der sich erst allmählich ausgleicht und einer Anpassung an das gesteigerte Sauerstoffbedürfnis des Körpers mit Hilfe intensiverer Atembewegungen Platz macht.

\section{Herz.}

Auf die Veränderungen im Kreislaufsystem möchte ich nicht eingehen, da das Material hier erheblich kleiner ist und weitergehende Schlüsse deshalb noch nicht zuläBt.

\section{B. Beobachtungen über das Verhalten bestimmter Gruppen von Lehrgangsteilnehmern.}

I. Die Lehrgangsteilnehmer, die vor Beginn des Kurses keine Leibesübungen getrieben hatten.

Es lag nahe, nach der Anamnese aus der Masse der Kursteilnehmer diejenigen abzusondern, die bisher nie Leibesübügen betrieben hatten, und ihre Entwicklung während des Lehrgangs in Gegensatz zu der der übrigen zu stellen. Dies waren in Kurs I Io von 83 (Durchschnittsgröße $172,3 \mathrm{~cm}$ ), in Kurs II 23 von 56 (Durchschnittsgröße I 72,0 cm). Es war von vorne herein anzunehmen, daß bei diesen Leuten die Einwirkung der durch die Leibesübungen veränderten Lebensweise sich deutlicher bemerkbar machen würde als bei denen, die bisher schon Sport oder Turnen, wenn auch vielleicht in beschränkterem Umfange als hier, getrieben hatten,. Diese Annahme fand auch bei beiden Lehrgängen ihre Bestätigung. Wir sehen sowohl beim Gewicht als auch bei den Muskelumfängen, daß die Zunahme dieser Sondergruppe (mit einer geringfügigen Ausnahme) am Schluß der Kurse teilweise erheb-

i) KNOLL, Ärztliche Unters, an Skiläufern. Jahrb. d. Schweiz. Skiverbandes. Bern I920.

lich die Zunahme derer übersteigt, die schon vorher Leibesübungen betrieben hatten. In Kurs II wird dies Verhalten allerdings nur dann deutlich, wenn die Leute mit 30 Jahren

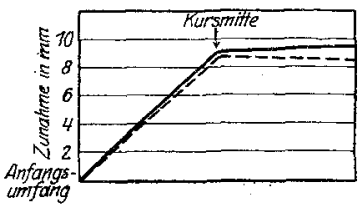

Kurs I:

Anfangswert $28,2 \mathrm{~cm}$

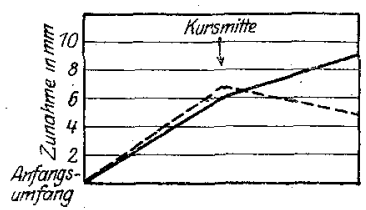

Kurs II:

- Anfangswert $28,5 \mathrm{~cm}$

"

Kurve B I: Oberarmumfang.

Kuisisten, d. früh. kein.Sport trieb.

" die früher Sport trieben

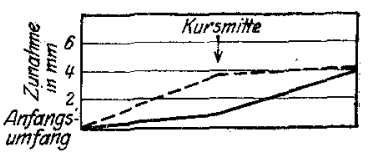

Kurs I: $\begin{array}{cr}\text { Anfangwert } & 49,5 \mathrm{~cm} \\ \ldots & \end{array}$

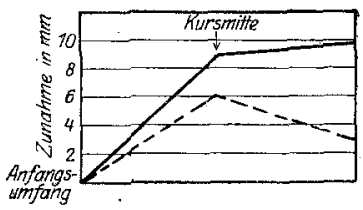

Kurs II: _.... Anfangswert $\} 48,6 \mathrm{~cm}$

Kurve B I: Oberschenkelumfang. Kursisten, d. früh. kein. Sport trieb. " die früher Sport trieben

und darüber ausgesondert werden, deren Verhalten offenbar andern Bedingungen unterworfen ist. In der "Sportgruppe" war keiner, in der ,Nicht-Sportgruppe" waren 9 solche Lehr-

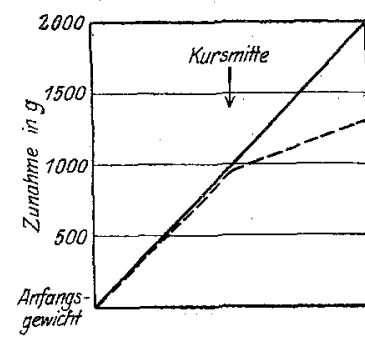

$$
\text { _... Anfangswert } 66,3 \mathrm{~kg}
$$
Kurve B I: Gewicht.

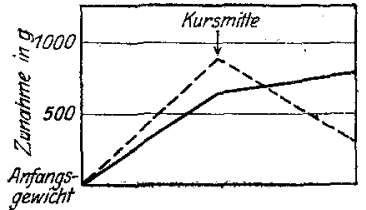

Kurs II Anfangswert $65,7 \mathrm{~kg}$ .... $\quad " \quad 66,0 \mathrm{~kg}$

Kursisten, d. früh. kein. Sport trieb. ..... Kursisten, die früher Sport trieben

gangsteilnehmer. Wenn man die beiden Gruppen nun vergleicht, so treten die Unterschiede sehr charakteristisch zu Tage. (Kurve B. I.). Am Kurs I nahm nur ein Beamter von 3o Jahren teil, was praktisch ohne Bedeutung ist.

Der Brustspielraum zeigte bei beiden Gruppen kein gesetzmäßiges Verhalten.

\section{Die Lehrgangsteilnehmer, die zu Beginn der Kurse ,unter- gewichtig" waren.}

Schon in den ersten Wochen beider Kurse war es aufgefallen, daß einzelne Leute besonders rapid an Gewicht zugenommen hatten. Bei näherer Untersuchung stellte es sich heraus, daB dies meist Leute von schlankem Wuchs waren. Man konnte ihren Körperbau im allgemeinen zwar nicht als asthenischen Typus, aber immerhin als grazil bezeichnen. Es wurden nun systematisch diejenigen ausgesondert, bei denen das Körpergewicht in erheblichem Mißverhältnis zu der Körperlänge stand, und zwar bildeten die eine Gruppe, bei denen das Körpergewicht zu Beginn der Kurse mehr als $7 \mathrm{~kg}$ hinter der Zentimeterzahl zurückblieb, um welche ihre Körperlänge roo $\mathrm{cm}$ überschritt. Dies waren in Kurs I 27 von 83 , in Kurs II 3 I von 56 . Teilnehmern. (Durchschnittsgröße I 72,7 bzw. I $74,4 \mathrm{~cm}$ ).

Die Entwicklung von Gewicht und Muskulatur war bei diesen Untergewichtigen graduell sehr von der der andern Lehrgangsteilnehmer verschieden. (Kurve B. II.). Ihre $\mathrm{Zu}$ nahmen betrugen oft ein vielfaches von denen der andern; auch waren die Rückschläge in der zweiten Hälfte der Kurse meist lange nicht so groß, so daß man zu der Feststellung 
berechtigt ist, daß die Einwirkung der intensiv betriebenen Leibesübungen auf diese untergewichtigen, grazil gebauten Menschen viel beträchtlicher war als auf die andern. Bei einer

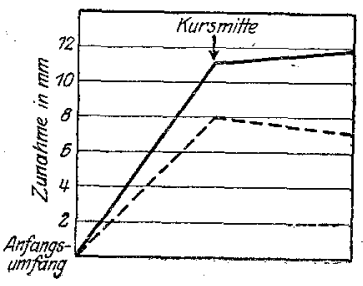

Kurs I
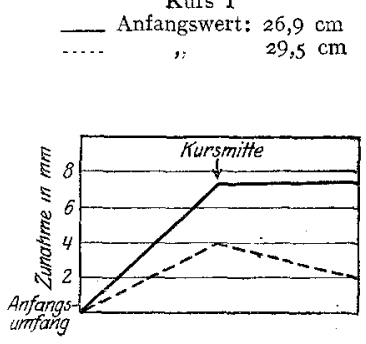

Kurs II

_. Anfangswert $27,3 \mathrm{~cm}$

Kurve B II: Oberarmumfang.

- Untergewichtige

-.... Nichtuntergewichtige

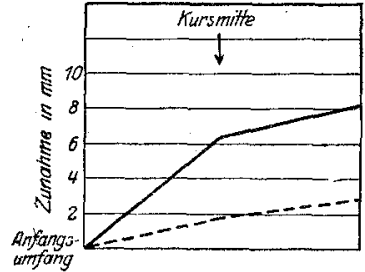

Kurs I __. Anfangswert $47,4 \mathrm{~cm}$

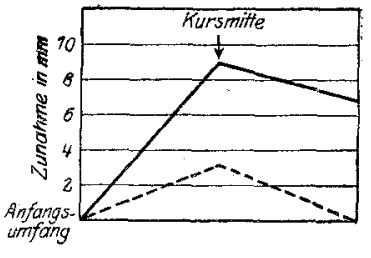

Kurs II - Anfangswert $47,3 \mathrm{~cm}$

Kurve B II: Oberschenkelumfans

_- Untergewichtige

-.... Nichtuntergewichtige ganzen Reihe von Untergewichtigen sprang das Gewicht bereits in den ersten Wochen ruckweise um mehrere $\mathrm{kg}$ in die Höhe - es wurde $z$. B. eine Zunahme von $4,5 \mathrm{~kg}$ in den

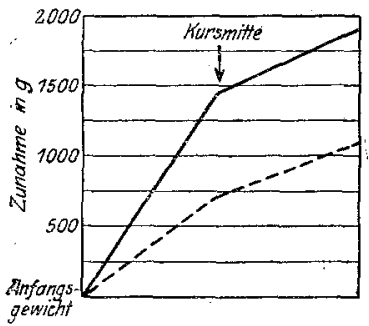
Kurs I
Anfangswert
62,4
$\ldots \ldots$$\quad \begin{gathered}\mathrm{kg} \\ 67,4\end{gathered}$ Kurve B II: Gewicht.

_._Untergewichtige

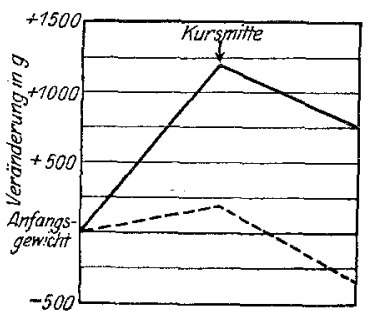

Kurs II: __. Anfangswert $63,0 \mathrm{~kg}$ . ersten 4 Wochen und eine solche von $5,4 \mathrm{~kg}$ innerhalb des ganzen Kurses beobachtet - so daß man den Eindruck hat daß dem Körper in diesen Fällen bisher der Wachstumsanreiz gefehlt habe, der ihm erst jetzt durch die körperliche Betätigung übermittelt worden sei. Es ist der Gedanke nicht von der Hand zu weisen, daß bei diesen Leuten eine gewisse Entwicklungshemmung als Folge mangelnder körperlicher Betätigung bestanden hat, die durch intensives Betreiben von Leibesübungen beseitigt wurde.

Es könnte hier der Einwand gemacht werden, daß bei den Untergewichtigen frühere Unterernährung eine Rolle gespielt habe. Um diese Fehlerquelle auszuschalten, wurden die Beamten über ihre früheren Ernährungsverhältnisse eingehend befragt, Dabei stellte sich heraus, daß sie zum allergrößten Teil über ihre bisherige Ernährung nicht zu klagen hatten.

Die Veränderung des Brustspielraums zeigte auch hier kein gesetzmäßiges Verhalten.

III. Die Lehrgangsteilnehmer, die bisher nur Leichtathletik und Rasenspiele, und diejenigen, die auch Schwerathletik, Boxen oder Turnen betrieben hatten.

Die Kursteilnehmer, die früher schon Leibesübungen getrieben hatten, hatten sich in recht verschiedenen Arten des Sportes betätigt. Es lag nun nahe, die bisherige Einwirkung dieser verschiedenen Sportzweige zu erforschen. Die absoluten Körpermaße konnten darüber keinen Aufschluß geben; dazu war die Zahl der Versuchspersonen zu gering. Dagegen konnte die Einwirkung, welche der sehr vielseitige und anstrengende Kurs auf ihren Körper auszuüben vermochte, als Maßstab für die Wirkungen der Leibesübungen angesehen werden, die bisher ihren Einfluß hatten geltend machen können.

Es wurden infolgedessen zwei Gruppen gebildet: die eine bestand aus denen, die bisher nur Leichtathletik - hauptsächlich Laufen - oder Rasenspiele - FuBball, Hockey und Rugby - oder beides betrieben hatten, also Sportarten, welche die untere Extremität sehr, die obere weniger beanspruchen; die andere Gruppe bestand aus Leuten, die bisher Gerätturnen, Boxen oder Schwerathletik - Ringen, Gewichtheben - betrieben hatten. Dies sind Ubungsarten, die sowohl an die untere wie die obere Extremität große Anforderungen stellen.

Ein gleichmäßiger Unterschied zwischen der Entwicklung beider Gruppen bestand jedoch nicht. Nach den Befunden in Kurs I schien es zunächst so, als ob die erste Gruppe erheblich mehr Muskelzuwachs insbesondere an der oberen Extremität aufzuweisen hätte als die letztere. Aber der Kurs II zeigte genau das umgekehrte Verhalten und es stellte sich heraus, daß die Unterschiede zwischen beiden Gruppen im wesentlichen durch die Zahl der Untergewichtigen bedingt waren, die darin enthalten war. Je mehr Untergewichtige sich in einer Gruppe befanden, um so größer wurde die $\mathrm{Zu}$ nahme dieser Gruppe an Gewicht und an Muskelumfängen. So waren in Kurs I in der Gruppe Leichtathleten und Rasenspieler $32 \%$, in der Gruppe Schwerathletik etc. $25 \%$ Untergewichtige. Die Ersteren nahmen mehr zu. In Kurs II dagegen waren in der Gruppe Leichtathletik etc. $53 \%$, in der Gruppe Schwerathletik dagegen $73 \%$ Untergewichtige. Hier nahmen die Letzteren mehr zu.

Ein wesentlicher Gewinn für unsere Betrachtungen läßt sich also aus diesen Ergebnissen nicht ziehen.

IV. Die Lehrgangsteilnehmer, die 30 Jahre und älter waren.

Schon früher wurde erwähnt, daß die Kursteilnehmer, die 3o Jahre und darüber alt waren, anders auf die durch das Betreiben von Leibesübungen geschaffene Milieuveränderung

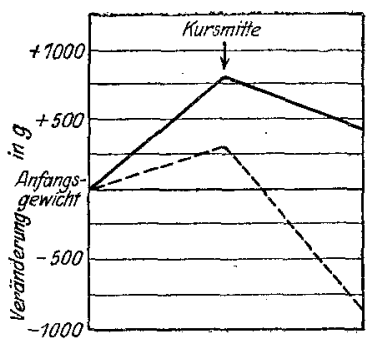

___ Anfangswert $65,9 \mathrm{~kg}$

-... $\quad 70, \mathrm{x} \mathrm{k}$

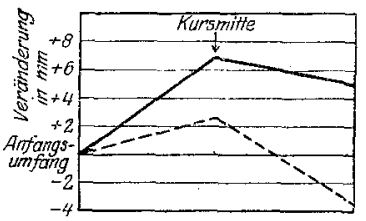

_. Anfangswert $48,6 \mathrm{~cm}$

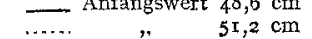

Kurve B IV: Oberschenkelumfang.

Kurven B IV :

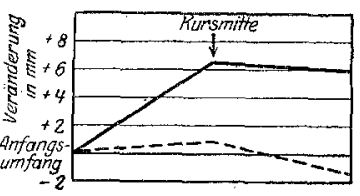

- Anfangswert 28,2 cm

" $29,9 \mathrm{~cm}$

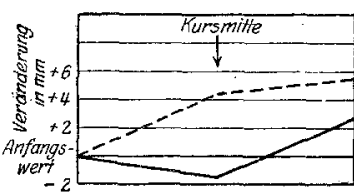

__ Anfangswert $7,6 \mathrm{~cm}$ Kurve B IV: Brustspielraum.

Kurs II unter 30 Jahren reagierten als die jüngeren. Aus der Kurve B. IV. erhellt, daß sowohl beim Gewicht wie den Muskelumfängen zwar eine anfängliche kurze Zunahme auftritt, die aber in der zweiten Kurshälfte von einem so überaus starken Abfall gefolgt ist, daß im Ganzen eine erhebliche Abnahme resultiert. Wir sehen im Kurs II - am Kurs I nahmen keine älteren Leute teil - bei diesen (9 von $5^{6}$ ) eine Gewichtsabnahme von $0,85 \mathrm{~kg}$ gegenüber einer Zunahme von $0,42 \mathrm{~kg}$ bei dem Rest 
des Kurses, beim Oberschenkel finden wir eine Abnahme von $0,36 \mathrm{~cm}$ gegenüber einer Zunahme von $0,5^{\circ} \mathrm{cm}$ und beim Oberarm eine Abnahme von o, I3 cm gegenüber einer Zunahme von $0,59 \mathrm{~cm}$. Das sind sehr große Unterschiede. $\mathrm{Zu}$ ihrer Deutung muß die Tatsache herangezogen werden, daß zu Beginn des Kurses das Fettpolster bei diesen älteren Kursteilnehmern im Durchschnitt erheblich besser war als bei den Jüngeren, was ja $z$. T. auch aus dem Verhältnis von Anfangsgewicht zur Körperlänge hervorgeht. Das ist wohl kein zufälliger Befund, sondern eher die Folge davon, daß mit zunehmendem Alter bei vielen Menschen die Neigung zum Ansatz von Fett, zunimmt. Wenn man dies in Betracht zieht, dann bedeutet unsere Beobachtung eine zum mindesten graduelle Veränderung gewisser Stoffwechselvorgänge mit fortschreitendem Alter bzw. bei großem Fettreichtum des Organismus. Es wäre denkbar, daß der gealterte Körper, dessen Stoffwechsel anders eingestellt ist, auf den Reiz intensiver körperlicher Tätigkeit anders reagiert als der junge und genötig $t$ ist, von seinen Reservestoffen zu verbrauchen. Bei der Kleinheit des Materials verbietet es sich jedoch, auf die weiteren Folgerungen einzugehen, welche diese Annahme ergeben würden.

Der Brustspielraum verhielt sich wie im Gesamtdurchschnitt der Kurse so auch hier umgekehrt wie Muskulatur und Gewicht; während diese hier abnahmen, nahm er stetig zu (Kurve B. IV.).

\section{Die Lehrgangsteilnehmer, die in der ersten Hälfte der Kurse verringerten Brustspielraum aufwiesen.}

Schon weiter oben sind gewisse Möglichkeiten für die Ursache der Verringerung des Brustspielraums zu Anfang der Kurse erörtert worden. Daß ein bestimmter Zusammenhang mit der Zunahme des Körpergewichts und der Muskulatur besteht, erscheint nach dem gleichmäßigen Verhalten in beiden Kursen sicher. Besonders deutlich mußte sich aber dieser Zusammenhang zeigen, wenn man diejenigen Kursteilnehmer zur Beobachtung aussonderte, die in der ersten Hälfte des Kurses eine Verminderung des Brustspielraums aufwiesen. Wenn diese dann eine besonders starke Muskulaturzunahme zeigten, dann war ein sehr enger Zusammenhang beider Erscheinungen deutlich gemacht.

Die gesonderte Berechnung der Veränderungen bei diesen Leuten wurde vorgenommen: es handelte sich in Kurs I um 38 , in Kurs II um 24 Personen. Es zeigte sich, daß tatsächlich diese Lehrgangsteilnehmer, wenigstens an Gewicht, mehr zugenommen hatten als die anderen (durchschnittlich I, I I kg gegen $0,85 \mathrm{~kg}$ in Kurs I und 1,20 gegen $0,42 \mathrm{~kg}$ in Kurs II). Aber bei den Muskelumfängen sehen wir nur in Kurs II das gleiche Bild. In Kurs I dagegen haben die Leute mit vermindertem Brustspielraum an Muskelumfang weniger zugenommen als die andern. Wir sehen also, daß nur in Bezug auf die Gewichtszunahme ein gleichmäßiges Verhalten vorliegt. Damit stimmt überein, daß umgekehrt bei der Gewichts. abnahme, die wir bei den älteren Kursteilnehmern feststellen konnten, eine Vergrößerung des Brustspielraumes erfolgte.

Da der Unterschied in der Gewichtszunahme der Leute mit verringertem und mit vergrößertem Brustspielraum zwar vorhanden, aber nicht in beiden Kursen gleich erheblich ist, so ist ein Zusammenhang zwischen beiden Erscheinungen wohl als bestehend anzunehmen; dieser Zusammenhang dürfte jedoch nur ein mittelbarer sein, da im andern Falle die festgestellten Unterschiede hätten größer sein müssen.

\section{Die Lehrgangsteilnehmer, die am Schluß des Lehrgangs an Gewicht abgenommen hatten.}

Die gesonderte Betrachtung dieser Gruppe ermöglicht es, den engen Zusammenhang zwischen der Zunahme der Muskelumfänge und dem Gewichtszuwachs näher zu verfolgen. Es nahmen in Kurs I I5, in Kurs II 2 I Personen an Gewicht ab. Diese hatten am Oberschenkel eine Veränderung von $-0,28 \mathrm{~cm}$ bzw. $-0,29 \mathrm{~cm}$ gegenüber $+0,6 \mathrm{I}$ und $+0,7^{8} \mathrm{~cm}$ bei dem Rest des Kurses zu verzeichnen, während am Oberarm eine Veränderung von $+0,49 \mathrm{~cm}$ bzw. $-0,05 \mathrm{~cm}$ einer Zunahme von $0,96 \mathrm{bzw} .0,8 \mathrm{r} \mathrm{cm}$ bei den andern gegenüberstand. Damit kann es als feststehend bezeichnet werden, daß die Gewichtszunahme in der Hauptsache auf das Wachstum der Muskulatur zurückgeht.

Schlußbetrachtung. Aus den hier geschilderten Beobachtungen geht hervor, daß der EinfluB der Leibesübungen auf den erwachsenen Körper ein ganz erheblicher ist. Dieser Einfluß ist im Durchschnitt für die körperliche Entwicklung ein durchaus förderlicher gewesen. Zieht man in Betracht, daß irgend welche ernsthaften Schädigungen während der beiden Kurse überhaupt nicht gesehen wurden, so muß man zu dem Schluß kommen, daß Sport und Turnen ein Faktor für đie körperliche Entwicklung unserer Jugend, unserer jungen Männer und damit unseres Volkes sein können, dessen Größe heute noch von der Mehrzahl der Zeitgenossen viel zu gering eingeschätzt wird. Deshalb sollten wir Ärzte in der Erkenntnis dieser Tatsache vorangehen und auf jede mögliche Weise, im Kleinen wie im Großen, die Förderung der Leibesübungen betreiben.

Im einzelnen sind die Ergebnisse der vorliegenden Untersuchungen folgende:

I. Unter dem Einfluß intensiv betriebener vielseitiger Leibesübungen nahm das Körpergewicht erwachsener junger Männer bei ausreichender Ernährung durchschnittlich erheblich zu.

2. Gleichzeitig ging eine deutliche durchschnittliche Zunahme der Umfänge von Oberarm und Oberschenkel vor sich, die in der Hauptsache auf Wachstum der Muskulatur zurückgeführt wird.

3. Die Gewichtszunahme ist mit Sicherheit auf dieses Wachstum der Muskulatur zurückzuführen.

4. Der Stoffansatz war bei niedriger AuGentemperatur erheblich geringer als bei hoher.

5. Der Brustspielraum nahm regelmäßig zu Beginn der körperlich anstrengenden Lebensweise etwas $a b$, um später stark anzusteigen. Diese Erscheinung kann vorläufig nicht sicher erklärt werden.

6. Diejenigen jungen Männer, die bisher keine Leibesübungen getrieben hatten, nahmen durchschnittlich mehr an Gewicht und Muskelumfängen $\mathrm{zu}$ als die andern.

7. Die grazil gebauten "Untergewichtigen“ unter den Beobachteten wiesen besonders starke und plötzliche Zunahmen an Gewicht und Muskelumfängen auf. Bei ihnen wirkte die ungewohnt starke körperliche Betätigung offenbar als besonders starker Wachstumsanreiz auf die Muskulatur.

8. Die wenigen zur Beobachtung gelangten Männer im Alter von 30 Jahren und darüber nahmen im Gegensatz zu den übrigen an Gewicht und Muskelumfängen ab. Hieraus darf vielleicht geschlossen werden, daß der Körper sich in vorgerückterem Alter gegenüber starker Beanspruchungen anders verhält als bè jüngeren Männern.

9. Wesentliche Verschiedenheiten in der Einwirkung verschiedener Arten körperlicher Betätigung auf die Muskulatur der oberen und der unteren Extremität, das Körpergewicht und den Brustspielraum ließen sich nicht nachweisen.

Io. Der stark fördernde Einfluß der Leibesübungen auf die Entwicklung des Körpers im Alter zwischen 24 und 30 Jahren hat sich auch durch die vorliegenden Untersuchungen bestätigt.

\section{NERVENLÄHMUNG UND NERVENREIZUNG IN IHRER BEDEUTUNG FÜR DIE ENTSTEHUNG TROPHISCHER GEWEBSVERÄNDERUNGEN.}

\author{
Von \\ Prof. Dr. FRITZ BRÜNING, \\ a. o. Professor der Chirurgie an der Universität Berlin.
}

Die landläufige Ansicht sah und sieht noch heute in den nach Nervenverletzungen und bei Nervenerkrankungen auitretenden vasomotorisch-trophischen Gewebsschäden eine Folge des Funktionsausfalls der betroffenen Nerven, also Folgen einer Nervenlähmung.

Diese Ansicht ist, wie ich zeigen werde, völlig unhaltbar, wir sind vielmehr verpflichtet die genannten Schäden zum größten Teil als die Folgen einer Nervenreizung anzusehen.

Angeregt durch eine Mitteilung von LERICHE kam mir der Gedanke, daß Beobachtungen, die ich an Nervenschußverletzungen im Kriege machte, sich nur dadurch erklären ließen, daß für die Entstehung trophischer Gewebsschäden nach Nervenverletzungen ein Reizzustand im Nerven verantwortlich gemacht werden müsse. 